\title{
Biomarkers in Neonatal Posthemorrhagic Hydrocephalus
}

\author{
Stephanie Merhar \\ Cincinnati Children's Hospital Medical Center, Cincinnati, Ohio, USA
}

\section{Key Words}

Posthemorrhagic hydrocephalus · Posthemorrhagic

ventricular dilation $\cdot$ Biomarker

\begin{abstract}
Posthemorrhagic hydrocephalus $(\mathrm{PHH})$ is a rare but serious outcome among premature babies in the NICU, with consequences including mortality and severe neurodevelopmental disabilities. The causes of PHH are still not entirely understood, and its prevention and treatment are controversial. Various cerebrospinal fluid biomarkers have been studied in infants with $\mathrm{PHH}$ in order to recognize the causes, diagnose brain injury, and predict neurodevelopmental outcomes. This systematic review summarizes studies on biomarkers of extracellular matrix activity, fibrinolysis/coagulation, hypoxia/cell death, and inflammation in the cerebrospinal fluid of infants with $\mathrm{PHH}$.

Copyright $\odot 2011$ S. Karger AG, Basel
\end{abstract}

\section{Introduction}

Despite the incidence declining in recent years [1], approximately $20 \%$ of very low birth weight infants develop the complication of intraventricular hemorrhage (IVH) during their NICU stay [2-4]. The most important direct complication of IVH is progressive enlargement of the ventricular system caused by accumulation of cerebrospinal fluid (CSF) in the ventricles, known as posthemorrhagic hydrocephalus (PHH). About 25\% of the babies with grade II-IV IVH will develop this complication [5]. Infants who develop PHH have worse neurodevelopmental outcomes than those with IVH without $\mathrm{PHH}$, especially if they are among the $40 \%$ of patients with $\mathrm{PHH}$ who require surgery for drainage of the hydrocephalus [5-8].

It is thought that acute $\mathrm{PHH}$ results from obstruction of the CSF spaces by blood or blood products. More slowly progressive $\mathrm{PHH}$ is likely caused by inflammatory changes around the CSF pathways, gliosis, and arachnoiditis [9]. Several factors are thought to contribute to brain injury in infants with IVH and PHH. Damage to the germinal matrix and hypoxia-ischemia, both possibly mediated by cytotoxins from blood products, are presumed to be the main mechanisms of injury after IVH [9]. Physical stress to the white matter due to ventricular distension is thought to cause brain injury in $\mathrm{PHH}$. Timing of intervention (CSF drainage by lumbar puncture, reservoir placement, or the more permanent ventriculoperitoneal shunt) in $\mathrm{PHH}$ remains controversial, as some infants will have spontaneous arrest of ventricular dilation [1]. The factors which cause the arrest of dilation and resolution of $\mathrm{PHH}$ in these infants are not known. Currently various clinical and radiographic criteria, such as rate of head growth, rate of increase of ventricular size on

\section{KARGER \\ Fax +4161306 1234 E-Mail karger@karger.ch} www.karger.com
(C) 2011 S. Karger AG, Basel 1661-7800/12/1011-0001\$38.00/0

Accessible online at: www.karger.com/neo
Stephanie Merhar, MD

Division of Neonatology, Cincinnati Children's Hospital

3333 Burnet Ave ML 7009

Cincinnati, OH 45229 (USA)

Tel. +1 513257 7749, E-Mail stephanie.merhar@cchmc.org 
head ultrasound, and changes in cerebral blood flow on Doppler ultrasound are used to determine when to intervene. However, there is currently no consensus as to when to intervene to prevent irreversible brain injury due to ventricular dilation while avoiding surgery in those babies who will have spontaneous arrest of $\mathrm{PHH}$.

Several authors have evaluated the CSF in patients with IVH and PHH to establish mechanisms of brain injury and possible targets for nonsurgical intervention. Although no CSF biomarkers are in clinical use in this population, biomarkers could potentially have several roles: determination of which infants with IVH are at risk for progression to $\mathrm{PHH}$, further elucidation of the mechanisms of white matter injury in $\mathrm{PHH}$ and at which point this injury begins to occur, and prediction of neurodevelopmental outcomes in infants with PHH. This article will review the literature on biomarkers in infants with $\mathrm{PHH}$ and determine whether any of these biomarkers are ready for routine clinical use.

\section{Methods}

\section{Data Sources}

The following electronic databases were searched: MEDLINE (PubMed), CINAHL Plus, and the Cochrane Library. A search was conducted using the following terms: posthemorrhagic hydrocephalus OR posthemorrhagic ventricular dilation OR posthemorrhagic ventricular dilatation AND biomarker OR cytokine OR genetic OR gene OR susceptibility. In addition to electronic database searches, reference lists of included studies were reviewed. Only English language studies and human studies were included.

\section{Inclusion Criteria}

The following inclusion criteria were applied: (1) hydrocephalus due to IVH or periventricular hemorrhagic infarction, and (2) premature infants ( $<37$ weeks gestation). Biomarkers had to be derived from CSF or blood, and thus studies that were exclusively neuroimaging based were excluded.

An initial literature search found only 10 papers, 4 of which were suitable for this review. Backwards reference search from these 4 articles and a review of references from relevant papers on $\mathrm{PHH}$ came up with an additional 25 articles, 17 of which were suitable for this review. Therefore, 21 papers in total met the inclusion criteria and were reviewed.

\section{Results}

The biomarkers described in $\mathrm{PHH}$ fall into four main categories: markers of collagen and extracellular matrix activity, markers of fibrinolysis and coagulation, markers of hypoxia and cell death, and markers of inflammation.

\section{Collagen and Extracellular Matrix Activity}

After IVH, multiple small blood clots are thought to form throughout the ventricles and CSF spaces [10]. CSF is normally reabsorbed through the arachnoid villi into the venous sinuses but may also be reabsorbed through small blood vessels in the ependyma. Both of these types of small channels could be blocked by blood clots. Transforming growth factor- $\beta_{1}$ (TGF- $\left.\beta_{1}\right)$ may be released into the CSF after IVH. This protein is known to upregulate the genes for extracellular matrix proteins such as collagen and fibronectin, and is normally involved in wound healing and scar formation [10]. There is some evidence from animal models that this protein is involved in hydrocephalus, as transgenic mice that overexpress TGF- $\beta_{1}$ in the central nervous system develop hydrocephalus [11].

Whitelaw et al. [12] attempted to investigate whether TGF- $\beta_{1}$ is involved in the pathogenesis of PHH by measuring concentrations of TGF- $\beta_{1}$ in 12 preterm controls with no IVH and 19 infants with PHH, 10 of whom eventually required a shunt. Infants with $\mathrm{PHH}$ had a significantly higher level of total TGF- $\beta_{1}$ than controls. They found that an initial TGF- $\beta_{1}$ CSF concentration over 6.5 $\mathrm{ng} / \mathrm{ml}$ had a sensitivity of $80 \%$ and a specificity of $78 \%$ to predict $\mathrm{PHH}$ that did not resolve on its own. $66 \%$ of the infants with $\mathrm{PHH}$ had activated TGF- $\beta_{1}$ present in the CSF, while none of the control infants had activated TGF$\beta_{1}$. The authors concluded that persistently elevated TGF$\beta_{1}$ may stimulate fibroblastic cells to produce extracellular matrix proteins in sufficient quality to lead to permanent blockage of CSF reabsorptive pathways.

In contrast, Heep et al. [13] did not find that TGF- $\beta_{1}$ differed in control infants and infants with $\mathrm{PHH}$. They did find, however, that there was a correlation between persistent TGF- $\beta_{1}$ and the occurrence of white matter injury in patients with $\mathrm{PHH}$. They concluded that persistent elevation of TGF- $\beta_{1}$ may be of prognostic value to indicate severity of brain injury in patients with $\mathrm{PHH}$. This same group again looked at TGF- $\beta_{1}$ [14], and found that TGF- $\beta_{1}$ was higher in patients with $\mathrm{PHH}$ than in patients with hydrocephalus from other reasons. They concluded that CSF malabsorption may contribute to the pathogenesis of $\mathrm{PHH}$ through the TGF- $\beta_{1}$ signaling cascade which regulates collagen synthesis and promotes cell proliferation.

Chow et al. [15] also looked at TGF- $\beta_{1}$, but included measurements of TGF- $\beta_{2}$ and chondroitin sulfate proteoglycans (CSPG) and their nitrated metabolites, NT-CSPG. TGF- $\beta_{2}$ has been found to decrease the proliferation of CNS precursors and to induce cell death of oligodendrocytes in vitro. CSPGs are the most prevalent proteogly- 
cans in the developing CNS and may contribute to the increase in extracellular matrix that could lead to scarring and obliteration of the CSF reabsorption pathways. These authors found that concentrations of CSF TGF- $\beta_{1}$, TGF$\beta_{2}$, CSPG, and NT-CSPG were increased in patients with $\mathrm{PHH}$ compared to preterm controls without $\mathrm{PHH}$. In contrast to the study by Whitelaw et al. [12], TGF- $\beta_{1}$ only weakly correlated with PHH. CSF NT-CSPG (but not the other 3 measured) was correlated with cystic white matter injury. The concentration of TGF- $\beta_{2}$ was $>20$ times greater in patients who required a shunt for $\mathrm{PHH}$ as compared to survivors who did not require a shunt or those without hydrocephalus. Bayley Mental Development (MDI) and Psychomotor Development Index (PDI) Scores were measured on $83 \%$ of surviving infants at a median adjusted age of 15 months. Concentration of TGF- $\beta_{2}$ was found to inversely correlate with MDI and PDI, while the concentration of NT-CSPG was found to inversely correlate with only the PDI. The authors concluded that although further studies are needed, CSPGs and TGF- $\beta_{2}$ may prove to be useful clinical prognostic tools in patients with $\mathrm{PHH}$.

Lipina et al. [16] followed 29 premature newborns with $\mathrm{PHH}$ and attempted to relate TGF- $\beta_{1}$ levels with the success of endoscopic third ventriculostomy (ETV) surgery. ETV is a surgical procedure in which neurosurgeons create an opening in the third ventricle, which allows CSF to bypass an obstructed aqueduct and leave the ventricular system. Theoretically, this surgery should only be effective in infants with $\mathrm{PHH}$ if obstruction (and not problems with reabsorption) is the main mechanism causing the hydrocephalus. In this group, ETV was performed in infants who continued to require ventricular taps to remove CSF and whose MRI scans revealed that the hydrocephalus had an obstructive component. The authors found that infants in whom the ETV failed (i.e. infants who required a shunt, called the 'hyporesorption group') had significantly higher values of TGF- $\beta_{1}$ than infants whose ETV procedures succeeded or who did not require ETV or shunt placement. Using a cutoff value of CSF TGF- $\beta_{1}$ of $2,396 \mathrm{pg} / \mathrm{ml}$, they found a sensitivity of $79 \%$ and specificity of $80 \%$ to predict 'hyporesorption'. The authors concluded that TGF- $\beta_{1}$ levels may be useful in deciding whether to perform ETV procedures in patients with $\mathrm{PHH}$.

To measure upregulation of extracellular matrix protein synthesis and fibroproliferative reactions in the CSF, Heep et al. [17] analyzed the concentrations of procollagen I C-propeptide (PICP) as a marker of activated collagen turnover in premature infants with $\mathrm{PHH}$, all of whom required shunt placement, and control infants with congenital hydrocephalus. Concentrations of PICP were higher in infants with $\mathrm{PHH}$ than in controls at all time points measured, but the concentrations were found to decline over time. The authors concluded that the increased collagen turnover is not a transient reaction immediately after injury, but is a more chronic process.

Okamoto et al. [18] studied matrix metalloproteinases (MMPs), which mediate the removal of extracellular matrix proteins. They measured the activity of inactive and active MMP-2 and MMP-9 in 3 infants with PHH, 3 controls with subarachnoid hemorrhage, and 3 normal controls who had lumbar punctures. Patients with $\mathrm{PHH}$ had increased activities of pro-MMP-9 (the inactive precursor form) compared to the 2 other groups. The patient who required a shunt had lower activity of pro-MMP-9 than the 2 patients who did not require a shunt for their $\mathrm{PHH}$. MMP-2 was not different between the 3 groups. The authors concluded that MMP-9 may play an important role in the arrest or resolution of ventricular dilation in $\mathrm{PHH}$.

Vascular endothelial growth factor (VEGF) is a glycoprotein that promotes angiogenesis and increases vascular permeability, which causes edema and breakdown of the blood-brain barrier [19]. Under hypoxic conditions, such as are hypothesized to occur in PHH, VEGF is upregulated in neurons, astrocytes, and microglia [19]. Heep et al. [13] found that VEGF was increased in patients with $\mathrm{PHH}$ as compared to patients with congenital hydrocephalus and controls. Koehne et al. [19] also looked at VEGF concentrations in patients with PHH (and other types of hydrocephalus, but patients with $\mathrm{PHH}$ were isolated), and found that the concentrations of VEGF were significantly increased as compared to controls without hydrocephalus. The authors concluded that the increased VEGF production in the CSF of children with $\mathrm{PHH}$ may be an adaptive response, but could have both detrimental and beneficial effects.

\section{Fibrinolysis and Coagulation}

As mentioned above, $\mathrm{PHH}$ is thought to result in part from multiple small clots that obstruct the flow of CSF through the ventricular system and arachnoid villi. The $25 \%$ [1] of infants who develop PHH after IVH could have impaired mechanisms of clearing these clots from the CSF as compared to those who do not develop PHH after similar IVH. Several authors have attempted to look at the endogenous fibrinolytic activity in infants with $\mathrm{PHH}$, and trials have been performed using exogenous fibrinolysis (tissue type plasminogen activator - tPA, streptokinase, and urokinase) to reduce the incidence of shunt placement after IVH; however, these trials have not been successful in reducing shunt surgery or death $[20,21]$. 
Plasminogen activator inhibitor-1 (PAI-1) is the primary inhibitor of endogenous plasminogen activators (tissue plasminogen activator and urokinase), and thus the primary inhibitor of fibrinolysis. Hansen et al. [22] measured PAI-1 levels in the CSF and plasma of newborns with and without PHH. They found that levels of PAI-1 were greatly elevated in patients with PHH compared to controls, and also higher in patients with $\mathrm{PHH}$ than in patients with IVH alone. In another study, this same group also found that PAI-1 was undetectable in control infants but was detectable in all but one of the $\mathrm{PHH}$ patients [23]. Fifteen out of $16 \mathrm{PHH}$ patients were treated with exogenous fibrinolytic agents (streptokinase, urokinase, or tPA), and the 6 patients who were treatment successes (i.e. who did not require shunt placement) had significantly lower levels of PAI-1 than the 10 patients who were treatment failures. The authors concluded that high levels of PAI-1 in the CSF are predictive of failure of intraventricular fibrinolytic therapy.

tPA was measured in the CSF of normal newborn infants and infants with $\mathrm{PHH}$ [24]. tPA was undetectable or at the limit of detection $(1 \mu \mathrm{g} / \mathrm{l})$ in controls, but ranged from 1.3 to $3.5 \mu \mathrm{g} / \mathrm{l}$ in the infants with PHH. The authors concluded that endogenous tPA may be part of the physiologic response to IVH. The same authors measured fibrinolytic activity by the fibrin plate method, a semiquantitative measurement which does not result in absolute units but can measure the presence of fibrinolytic activity in the CSF of control infants and infants with IVH [25]. All samples taken less than 17 days after diagnosis of IVH did not show fibrinolytic activity, but all but one of the samples taken between 17 and 60 days after IVH did show fibrinolytic activity. No significant differences were found in the infants who developed $\mathrm{PHH}$ versus the infants who had IVH but did not develop PHH. The authors concluded that the neonate has capacity for endogenous fibrinolysis after IVH, but that this capacity may not always be adequate to clear large amounts of clot. Cross-linked fibrin degradation products (FDP) were measured in the CSF of 4 infants with IVH, 5 infants with IVH that progressed to $\mathrm{PHH}$, and 5 controls [26]. Infants with IVH (median $315 \mathrm{ng} / \mathrm{ml}$ ) had higher concentrations of FDP than controls (median $102 \mathrm{ng} / \mathrm{ml}$ ), and infants with $\mathrm{PHH}$ (median 1,000 ng/ml) had even higher concentrations than those with IVH alone.

\section{Hypoxia and Cell Death}

The mechanism of brain injury in $\mathrm{PHH}$ is uncertain, but hydrocephalus is thought to produce compression of the periventricular white matter and eventually a reduc- tion in circulation, leading to hypoxia/ischemia and then cell death [9]. Therefore, several researchers have investigated markers of hypoxia and cell death in the CSF of patients with $\mathrm{PHH}$.

Thrombopoietin (TPO) is a hematopoietic growth factor expressed in the central nervous system which may have proapoptotic effects in the brain [27]. Reinhold et al. [27] measured TPO in neonates with $\mathrm{PHH}$ without infection, $\mathrm{PHH}$ with bacterial infection/sepsis, control infants, and infants without PHH but with bacterial infection/sepsis or meningitis. TPO was detectable in the CSF of 6 of 15 neonates with bacterial infection/sepsis, 5 of 9 neonates with PHH, 3 of 4 neonates with $\mathrm{PHH}$ plus bacterial infection/sepsis, but not in controls. The authors concluded that TPO might act as an acute phase reactant in the brain, and that a significant amount of TPO is produced in the brain, although they admit that their findings are heterogeneous. Erythropoietin (EPO) is another hematopoietic growth factor that acts as a cytokine in the brain and increases strongly under hypoxic conditions. Eighteen patients with PHH had EPO levels measured, and the levels were not significantly different from controls [19]. The authors hypothesized that even if EPO production is increased in infants with hydrocephalus, the EPO may not have reached the CSF.

Hypoxanthine is the end product of purine metabolism and is a marker for anaerobic metabolism and thus hypoxia/ischemia. In normal infants, hypoxanthine is absent in the CSF or present only in very low concentrations [28]. In infants with progressive ventricular enlargement, hypoxanthine concentrations were elevated, suggesting the presence of anaerobic metabolism in the brain tissue [28]. As the hydrocephalus was treated with serial lumbar punctures or ventriculoperitoneal shunt, the hypoxanthine concentration decreased. When hydrocephalus did not resolve following intervention, the hypoxanthine concentrations remained elevated or increased. The authors speculated that hypoxanthine concentrations could be used to detect tissue compromise and thus to time intervention in $\mathrm{PHH}$.

Reactive oxygen species are thought to play an important role in the pathogenesis of periventricular white matter injury in premature neonates. The hydroxyl radical is a highly reactive oxygen species that is generated from hydrogen peroxide in a reaction that requires a transitional metal such as non-protein-bound iron (NPBI) [29]. NPBI was present in CSF of 15/20 infants with PHH and $0 / 10$ control infants. There was no difference in NPBI levels between infants with and without need for shunting, 
with and without white matter lesions, or between infants with normal outcome, motor disability, or multiple disability. The authors hypothesized that NPBI could be released from heme catabolism from the degrading blood in the CSF or released from the iron storage protein ferritin. The authors discussed that the process by which NPBI exerts its harmful effect is not likely dose dependent, and that imprecise timing of sampling may be why no correlation was found between the absolute level of NPBI and outcome or between NPBI and the degree of white matter damage.

Animal studies in hydrocephalus have shown that damage of neurons, axons, and oligodendrocytes is associated with apoptotic cell death [30]. Apoptosis is partially regulated by the Fas/Fas ligand system and a protease known as caspase 3. Felderhoff-Mueser et al. [30] found that soluble Fas (sFas) was higher in patients with $\mathrm{PHH}$ and non-hemorrhagic hydrocephalus than controls. sFas concentrations were highest in a subgroup of 8 patients with $\mathrm{PHH}$ who developed white matter injury. sFas ligand and caspase 3 did not differ between the infants with hydrocephalus and the control infants. The authors concluded that apoptosis is occurring in the brains of infants with hydrocephalus. They hypothesized that sFasL and caspase 3 were absent because hydrocephalus is a more chronic than acute process, and also the levels of these proteins may be too low in the CSF of infants with $\mathrm{PHH}$ to be detected by their methods.

Whitelaw et al. [31] attempted to evaluate white matter injury in infants with $\mathrm{PHH}$ by investigating levels of the brain-specific proteins neurofilament triplet protein, glial fibrillary acid protein, and S-100. CSF concentrations of all 3 proteins were found to be 4-200 times higher in patients with $\mathrm{PHH}$ than in control infants. All 3 proteins were also higher in infants with $\mathrm{PHH}$ and parenchymal brain lesions than in those with $\mathrm{PHH}$ alone, and higher in infants with $\mathrm{PHH}$ who died or were disabled at 12 months of age than in those with $\mathrm{PHH}$ who were alive without disability at 12 months. The authors concluded that elevation of these brain-specific proteins provides evidence of periventricular white matter damage in infants with $\mathrm{PHH}$.

\section{Inflammation and Miscellaneous}

Many infants with IVH and PHH develop white matter injury, which is thought to be mediated in part by damage from proinflammatory cytokines. Savman et al. [32] evaluated the CSF of infants with $\mathrm{PHH}$ and attempted to correlate the concentrations of cytokines with ultra- sound evidence of periventricular white matter damage, neurodevelopmental outcomes, and need for shunting. They found high levels of proinflammatory cytokines (tumor necrosis factor- $\alpha$, interleukin (IL)-1 $\beta$, IL- 6 , and IL-8) in the CSF from preterm infants with PHH compared with preterm controls. However, no correlation was found between cytokine levels and white matter lesions or outcome. The authors speculated that the high levels of cytokines could be a reaction to the hemorrhage and not actually reflect the severity of the brain damage. They also feel that error may have been introduced because the CSF was sampled so long after the initial insult (at a median of 2 weeks of life).

Schmitz et al. [33] also looked at proinflammatory cytokines in infants with $\mathrm{PHH}$ and attempted to correlate the levels with patients who developed white matter injury. The first CSF sample was obtained at reservoir insertion, and the second sample was obtained at shunt insertion. Compared to controls without $\mathrm{PHH}$, infants with $\mathrm{PHH}$ with and without white matter injury had increased IL-1 $\beta$. Infants with PHH and no white matter injury had increased IL-18 in both samples compared to controls, and patients with $\mathrm{PHH}$ and white matter injury had elevated IL-18 compared to controls only in sample 2. Finally, interferon- $\gamma(\mathrm{IFN}-\gamma)$ was increased only in sample 1 and only in patients with $\mathrm{PHH}$ and white matter injury, not in controls or in patients with $\mathrm{PHH}$ without white matter injury. This is particularly important because IFN- $\gamma$ is thought to possibly be detrimental to the myelination process in the developing brain [33].

Trauma, hypoxia, infection, and the presence of blood are known to promote synthesis of eicosanoids (the family consisting of prostaglandins, prostacyclins, thromboxanes, and leukotrienes) [34]. Eicosanoids also are known to play a role in edema, and thromboxane $B_{2}$ is known to vasoconstrict cerebral arteries [34]. White et al. [34] studied eicosanoids in the CSF of patients with $\mathrm{PHH}$. Most of the 11 patients with $\mathrm{PHH}$ had at least one of the eicosanoids studied, with $70 \%$ of the sample containing leukotrienes. The CSF values of eicosanoids decreased with time in infants who had multiple CSF samples taken. 5 of the 6 infants who required a shunt had both thromboxane $B_{2}$ and leukotrienes, whereas only 1 of the 5 infants who did not require a shunt had both of these eicosanoids. The authors conclude that eicosanoids are found in most infants with $\mathrm{PHH}$, and thromboxane $\mathrm{B}_{2}$ and leukotrienes may be valuable to predict shunt requirement. 


\section{Discussion}

IVH and its consequence, $\mathrm{PHH}$, are some of the most feared complications of prematurity due to their association with poor outcomes (death and neurodevelopmental disability in later life). For this reason, there has been much interest in studying the predictors of development of PHH after IVH, the development of brain injury in already established $\mathrm{PHH}$ in order to time interventions, and predictors of prognosis (shunt placement and neurodevelopmental impairment) in order to provide information to families. An advantage in studying this disease is that CSF, which can be a direct indicator of what is happening in the brain, is readily available in many of these patients because CSF removal is often required for therapeutic reasons. However, it seems that some researchers have looked at substances in the CSF of patients with PHH simply because CSF is available, without a real hypothesis in mind. In addition, because CSF samples were taken for clinical reasons, serum levels of biomarkers were usually not studied, which makes it difficult to determine whether CSF concentrations of biomarkers were secondary to brain production or to leakage across the blood-brain barrier.

$\mathrm{PHH}$ is a rare outcome, and sample sizes in most of the studies have been small, which limits the power to detect differences between groups. In addition, many of the biomarkers were evaluated in only one study, which makes it difficult to comment on external validity of the results. Because there is no true consensus definition of what constitutes $\mathrm{PHH}$, some studies were not clear as to how they defined $\mathrm{PHH}$ beyond 'dilation of ventricles after IVH'. Minimal to moderate dilation of ventricles can be due to atrophy of white matter as well as increased pressure from hydrocephalus, so it is important to specify parameters of ventricular size and of patient history in defining $\mathrm{PHH}$. The patients in these studies were sampled at different times in the course of $\mathrm{PHH}$, and as such, even studies that looked at the same biomarkers may not be comparable. Some studies looked prospectively at infants early in the course of IVH and $\mathrm{PHH}$ when the ventricles were initially found to be dilated, while some studies looked at infants much later in the course (after shunt placement).

However, despite the many limitations of these studies, several markers may warrant validation in larger studies. TGF- $\beta_{1}$ and TGF- $\beta_{2}$ have the most biologic plausibility as markers of extracellular matrix upregulation and thus whether patients will develop hydrocephalus that requires surgical intervention. Studies on fibrinolyt- ic activity are interesting in the context of possible treatment with exogenous fibrinolytic agents, but unfortunately most studies of these agents in babies with $\mathrm{PHH}$ have not resulted in improved outcomes. However, a recent study on long-term outcomes of patients with $\mathrm{PHH}$ who received CSF drainage, irrigation, and fibrinolytic therapy found that infants in this group were less likely to have died or be severely disabled at age 2 than infants who received standard care [35]. The best use of a biomarker in this population would seem to be a marker to show when brain cells are being irreversibly damaged and thus when surgical intervention should take place. Markers of apoptosis and hypoxia would seem to be the most promising biomarkers for future studies in this arena.

The only biomarker that may be ready for routine clinical use to determine which infants with $\mathrm{PHH}$ have obstructive hydrocephalus and may benefit from an ETV procedure is TGF- $\beta_{1}$. Lipina et al. [16] published the sensitivity and specificity of a particular TGF- $\beta_{1}$ cutoff point for this decision. An ideal future study on the topic of $\mathrm{PHH}$ would be prospective and large scale and would include several CSF and blood biomarkers at multiple time points in infants with grades II-IV IVH. Infants would then be followed to determine development of $\mathrm{PHH}$ (defined using predetermined ultrasound criteria), necessity of surgical intervention, and neurodevelopmental outcomes.

\section{References}

1 Volpe JJ: Neurology of the Newborn, ed 5. Amsterdam, Elsevier Health Sciences, 2008.

2 Batton DG, Holtrop P, DeWitte D, Pryce C, Roberts C: Current gestational age-related incidence of major intraventricular hemorrhage. J Pediatr 1994;125:623-625.

3 Heuchan AM, Evans N, Henderson Smart DJ, Simpson JM: Perinatal risk factors for major intraventricular haemorrhage in the Australian and New Zealand Neonatal Network, 1995-97. Arch Dis Child Fetal Neonatal Ed 2002;86:F86-F90.

4 Sheth RD: Trends in incidence and severity of intraventricular hemorrhage. J Child Neurol 1998;13:261-264.

5 Murphy BP, Inder TE, Rooks V, Taylor GA, Anderson NJ, Mogridge N, et al: Posthaemorrhagic ventricular dilatation in the premature infant: natural history and predictors of outcome. Arch Dis Child Fetal Neonatal Ed 2002;87:F37-F41.

-6 Allan WC, Dransfield DA, Tito AM: Ventricular dilation following periventricular-intraventricular hemorrhage: outcome at age 1 year. Pediatrics 1984;73:158-162. 
7 Davis SL, Tooley WH, Hunt JV: Developmental outcome following posthemorrhagic hydrocephalus in preterm infants. Comparison of twins discordant for hydrocephalus. Am J Dis Child 1987;141:1170-1174.

$\checkmark 8$ Sasidharan P, Marquez E, Dizon E, Sridhar CV: Developmental outcome of infants with severe intracranial-intraventricular hemorrhage and hydrocephalus with and without ventriculoperitoneal shunt. Childs Nerv Syst 1986;2:149-152.

$\checkmark 9$ du Plessis AJ: Posthemorrhagic hydrocephalus and brain injury in the preterm infant: dilemmas in diagnosis and management. Semin Pediatr Neurol 1998;5:161-179.

$>10$ Whitelaw A: Intraventricular haemorrhage and posthaemorrhagic hydrocephalus: pathogenesis, prevention and future interventions. Semin Neonatol 2001;6:135-146.

- 11 Galbreath E, Kim SJ, Park K, Brenner M, Messing A: Overexpression of TGF-beta 1 in the central nervous system of transgenic mice results in hydrocephalus. J Neuropathol Exp Neurol 1995;54:339-349.

12 Whitelaw A, Christie S, Pople I: Transforming growth factor-betal: a possible signal molecule for posthemorrhagic hydrocephalus? Pediatr Res 1999;46:576-580.

-13 Heep A, Stoffel-Wagner B, Bartmann P, Benseler S, Schaller C, Groneck P, et al: Vascular endothelial growth factor and transforming growth factor-betal are highly expressed in the cerebrospinal fluid of premature infants with posthemorrhagic hydrocephalus. Pediatr Res 2004;56:768774.

- 14 Heep A, Bartmann P, Stoffel-Wagner B, Bos A, Hoving E, Brouwer O, et al: Cerebrospinal fluid obstruction and malabsorption in human neonatal hydrocephaly. Childs Nerv Syst 2006;22:1249-1255.

15 Chow LC, Soliman A, Zandian M, Danielpour M, Krueger RC Jr: Accumulation of transforming growth factor-beta2 and nitrated chondroitin sulfate proteoglycans in cerebrospinal fluid correlates with poor neurologic outcome in preterm hydrocephalus. Biol Neonate 2005;88:1-11.
16 Lipina R, Reguli S, Novackova L, Podesvova $\mathrm{H}$, Brichtova E: Relation between TGF-beta 1 levels in cerebrospinal fluid and ETV outcome in premature newborns with posthemorrhagic hydrocephalus. Childs Nerv Syst 2010;26:333-341.

17 Heep A, Stoffel-Wagner B, Soditt V, Aring C, Groneck P, Bartmann P: Procollagen I Cpropeptide in the cerebrospinal fluid of neonates with posthaemorrhagic hydrocephalus. Arch Dis Child Fetal Neonatal Ed 2002; 87:F34-F36

18 Okamoto T, Takahashi S, Nakamura E, Nagaya K, Hayashi T, Shirai M, et al: Matrix metalloproteinases in infants with posthemorrhagic hydrocephalus. Early Hum Dev 2008; 84:137-139.

19 Koehne P, Hochhaus F, Felderhoff-Mueser U, Ring-Mrozik E, Obladen M, Buhrer C: Vascular endothelial growth factor and erythropoietin concentrations in cerebrospinal fluid of children with hydrocephalus. Childs Nerv Syst 2002;18:137-141.

20 Whitelaw A, Odd DE: Intraventricular streptokinase after intraventricular hemorrhage in newborn infants. Cochrane Database Syst Rev 2007; CD000498.

21 Whitelaw A, Evans D, Carter M, Thoresen M, Wroblewska J, Mandera M, et al: Randomized clinical trial of prevention of hydrocephalus after intraventricular hemorrhage in preterm infants: brain-washing versus tapping fluid. Pediatrics 2007; 119:e1071-e1078.

22 Hansen AR, Lapp C, Brugnara C: Plasminogen activator inhibitor-1: defining characteristics in the cerebrospinal fluid of newborns. J Pediatr 2000;137:132-134.

23 Hansen A, Whitelaw A, Lapp C, Brugnara C: Cerebrospinal fluid plasminogen activator inhibitor-1: a prognostic factor in posthaemorrhagic hydrocephalus. Acta Paediatr 1997; 86:995-998.

24 Whitelaw A, Mowinckel MC, Fellman V, Abildgaard U: Endogenous tissue plasminogen activator in neonatal cerebrospinal fluid. Eur J Pediatr 1996;155:117-119.

25 Whitelaw A: Endogenous fibrinolysis in neonatal cerebrospinal fluid. Eur J Pediatr 1993; 152:928-930.

-26 Whitelaw A, Creighton L, Gaffney P: Fibrinolysis in cerebrospinal fluid after intraventricular haemorrhage. Arch Dis Child 1991; 66:808-809.
27 Reinhold A, Zhang J, Gessner R, FelderhoffMueser U, Obladen M, Dame C: High thrombopoietin concentrations in the cerebrospinal fluid of neonates with sepsis and intraventricular hemorrhage may contribute to brain damage. J Interferon Cytokine Res 2007;27:137-145.

28 Bejar R, Saugstad OD, James H, Gluck L: Increased hypoxanthine concentrations in cerebrospinal fluid of infants with hydrocephalus. J Pediatr 1983;103:44-48.

-29 Savman K, Nilsson UA, Blennow M, Kjellmer I, Whitelaw A: Non-protein-bound iron is elevated in cerebrospinal fluid from preterm infants with posthemorrhagic ventricular dilatation. Pediatr Res 2001;49:208-212.

$>30$ Felderhoff-Mueser U, Buhrer C, Groneck P, Obladen M, Bartmann P, Heep A: Soluble Fas (CD95/Apo-1), soluble Fas ligand, and activated caspase 3 in the cerebrospinal fluid of infants with posthemorrhagic and nonhemorrhagic hydrocephalus. Pediatr Res 2003;54:659-664.

-31 Whitelaw A, Rosengren L, Blennow M: Brain specific proteins in posthaemorrhagic ventricular dilatation. Arch Dis Child Fetal Neonatal Ed 2001;84:F90-F91.

32 Savman K, Blennow M, Hagberg H, Tarkowski E, Thoresen M, Whitelaw A: Cytokine response in cerebrospinal fluid from preterm infants with posthaemorrhagic ventricular dilatation. Acta Paediatr 2002;91: 1357-1363.

33 Schmitz T, Heep A, Groenendaal F, Huseman D, Kie S, Bartmann P, et al: Interleukin1beta, interleukin-18, and interferon-gamma expression in the cerebrospinal fluid of premature infants with posthemorrhagic hydrocephalus - markers of white matter damage? Pediatr Res 2007;61:722-726.

34 White RP, Leffler CW, Bada HS: Eicosanoid levels in CSF of premature infants with posthemorrhagic hydrocephalus. Am J Med Sci 1990;299:230-235.

35 Whitelaw A, Jary S, Kmita G, Wroblewska J, Musialik-Swietlinska E, Mandera M, et al: Randomized trial of drainage, irrigation and fibrinolytic therapy for premature infants with posthemorrhagic ventricular dilatation: developmental outcome at 2 years. Pediatrics 2010;125:e852-e858. 